Originales

\title{
Expresión de CD44v6 en carcinomas escamosos de pulmón. Asociación con altas concentraciones de EGFR, proteína erbB2 y elevada proliferación celular
}

\author{
A. Ruibal*, J. Barandela*, V. Pubul*, J. Rodríguez**
}

\section{Resumen}

- Propósito: el CD44v6 es una isoforma variante del CD44 involucrada en la diseminación metastática de ciertos tumores. Nosotros hemos querido analizar su expresión en carcinomas escamosos de pulmón y correlacionarla con otros parámetros clínico-biológicos de utilidad clínica.

- Material y método: el CD44v6 fue determinado mediante un EIA (Bender Diagnostics. Austria), cuyo dintel de positividad fue establecido en $5 \mathrm{ng} / \mathrm{mg}$ prot. En las membranas celulares determinamos, asimismo, las concentraciones del receptor del factor de crecimiento epidérmico (EGFR) y proteína erbB2. También se ha tenido presente el estadio clínico, grado histológico (GH), ploidía y fase de síntesis celular (FS).

- Resultados: en los carcinomas escamosos de pulmón, la positividad para el CD44v6 fue apreciada en 76 de los 95 casos (80\%). Los tumores CD44v6 positivos presentaron mayores concentraciones de EGFR (p:0,007), proteína erbB2 (p:0,056) y valores globales de FS (p:0,011). Asimismo, cursaron más frecuentemente con $\mathrm{FS}+(>15,4 \%)$ (p:0,041).

- Conclusiones: los resultados anteriores nos inducen a las siguientes consideraciones: 1) la expresión de CD44v6 se observa en un alto porcentaje de los carcinomas escamosos de pulmón; 2) Su asociación con altas concentraciones de EGFR, proteína erbB2 y una proliferación celular elevada sugieren que pueda reflejar un peor comportamiento y evolución de estos tumores.

Palabras clave:

CD44v6. Carcinoma escamoso de pulmón. EGFR. Proliferación.

Oncología, 2004; 27 (8):499-504

* Servicio de Medicina Nuclear

Hospital Clínico. Complejo Hospitalario Universitario

Santiago de Compostela

** Servicio de Cirugía Torácica

Hospital Central de Asturias. Oviedo 


\section{Summary}

- Purpose: CD44v6 is an adhesion molecule implicated in tumor invasion. We studied its expressión in squamous cell carcinomas and its correlation with other clinico-biological parameters.

- Material and methods: CD44v6 levels were measured in 95 cases of squamous lung carcinoma (SLC) using an enzyme immunoassay kit (EIA, Bender Diagnostics, Austria). Likewise, we determined the concentration of the epidermal growth factor receptor (EGFR) and erbB2 oncoprotein on the cell surface. The clinical stage, histological grade, ploidy and phase of cellular synthesis (SP) were also taken into account.

- Results: The CD44v6 expression ( $>5 \mathrm{ng} / \mathrm{mg}$ prot.) was observed in 76 out of the 95 cases of SLC. The CD44v6-positive tumors showed higher concentrations of EGFR ( $p=0.007$ ) and erbB2 protein $(p=0.056)$, and greater SP global values $(p=0.011)$ than the CD44v6-negative tumors. Positive tumors were more frequently SP+ $(>15 \%)(p=0.041)$.

- Conclusions: These results led us to the following conclusions: 1) The expression of CD44v6 in SLC is very frequent. 2) Its association with higher concentrations of EGFR and erbB2, as well as the greater cell proliferation suggests that it could reflect a poorer outcome. 3) Further studies are required to establish the clinical usefulness of CD44v6 expression in this subtype of lung tumors.

Key words: CD44v6. Squamous lung carcinoma. EGFR. ErbB2. Cell proliferation.

\section{Introducción}

El CD44 es una familia de moléculas involucradas en las interrelaciones célula-célula y célula-matriz extracelular. Existe una forma estándar (CD44s) y otras variantes (CD44v) consecuencia de modificaciones post-translacionales y splicing alternativos, muchas de las cuales se consideran marcadores de metástasis e indicadores pronósticos en determinados tumores.

La isoforma variante v6 (CD44v6) actúa como molécula de adhesión en los macrófagos y células similares, lo cual sugiere que su concentración sérica se pueda incrementar en situaciones no tumorales'. En el campo oncológico, su interés clínico varía según cual sea el tumor estudiado, habiéndose demostrado su valor pronóstico en los de cabeza y cuello ${ }^{2}$, diferenciados gástricos ${ }^{3}$, cérvix uterino $0^{4-5}$, linfomas no Hodgkin y leucemias mieloides ${ }^{6}$ y sarcomas de partes blandas ${ }^{7}$.

A nivel sérico su determinación puede ser de interés como indicador pronóstico en el cáncer gástrico tipo difuso ${ }^{8}$, colo-rectales ${ }^{9}$ y pancreáticos ${ }^{10}$. Asimismo, puede ser reflejo de un peor pronóstico y reducida respuesta a la hormono y quimioterapia en el cáncer de mama $^{1}$.

A nivel del árbol respiratorio, se ha observado que la expresión de CD44 por el epitelio bronquial se incrementa tras el daño tisular y puede ser el exponente de la motilidad celular, dediferenciación o una adhesión alterada ${ }^{2}$ y lo mismo ocurre con las isoformas va- riantes $^{13}$. Así, se puede constatar expresión del CD44v6 en el epitelio pulmonar normal ( preferentemente neumocitos tipo II) donde parece jugar un papel importante en el mantenimiento de la arquitectura tisu$\operatorname{lar}^{14}$. La postividad inmunohistoquímica es más frecuente en los tumores escamosos y bronquioalveolares ${ }^{15}$; en los tumores neuroendocrinos de pulmón se correlaciona inversamente con la agresividad ${ }^{16}$, mientras que en los microcíticos la positividad parece asociarse con la afectación ganglionar ${ }^{17,18}$.

Nosotros, en este trabajo, hemos querido estudiar la expresión inmunológica del CD44v6 en los carcinomas escamosos de pulmón y correlacionarla con otros parámetros clínico-biológicos que ayudaran a comprender su interés fisiopatológico y posible utilidad práctica.

\section{Material y métodos}

El grupo estudio incluyó 95 muestras tisulares tumorales de otros tantos pacientes afectos de carcinoma escamosos de pulmón, sin tratamiento previo, de edades comprendidas entre 36 y 79 años (mediana 64), de los cuales 72 eran varones. Asimismo, en 38 casos obtuvimos una muestra de tejido pulmonar aparentemente normal. Tras su obtención en el quirófano y transporte en un contenedor frío, las muestras fueron separadas de sus componentes grasos y almacenadas 

Distribución de los valores (intervalo, mediana y porcentaje de positividades) de los diferentes parámetros clínico-biológicos en carcinomas escamosos de pulmón clasificados en función de la positividad (>5 ng $/ \mathrm{mg}$ prot.) para el CD44v6

\begin{tabular}{|c|c|c|c|c|c|}
\hline \multirow[b]{2}{*}{ Parámetro } & \multicolumn{2}{|c|}{$>5$ (76 cases $)$} & \multicolumn{2}{|c|}{$</=5$ (19 cases) } & \multirow[b]{2}{*}{$\mathbf{p}$} \\
\hline & Intervalo & Mediana & Intervalo & Mediana & \\
\hline EGFR* & $3,1-325$ & 38,7 & $1-394$ & 21,3 & 0,007 \\
\hline ErbB2** & $219-77895$ & 1969 & $246-7848$ & 1532 & 0,056 \\
\hline $\mathrm{FS}++$ & $1,2-51,8$ & 16 & $1,8-28,9$ & 9,9 & 0,011 \\
\hline Tamaño++++ & $1-12$ & 4 & $2,5-7$ & 3,5 & ns \\
\hline GHI & \multicolumn{2}{|c|}{$6 / 76$} & \multicolumn{2}{|c|}{$3 / 19$} & ns \\
\hline GH2 & \multirow{2}{*}{\multicolumn{2}{|c|}{$\begin{array}{l}44 / 76 \\
26 / 76\end{array}$}} & \multicolumn{2}{|c|}{$8 / 19$} & ns \\
\hline GH3 & & & \multirow{2}{*}{\multicolumn{2}{|c|}{$\begin{array}{l}8 / 19 \\
15 / 19\end{array}$}} & ns \\
\hline ESTADIO I & \multicolumn{2}{|c|}{$4 / 76$} & & & ns \\
\hline ESTADIO ॥ & \multicolumn{2}{|c|}{$7 / 76$} & $1 / 19$ & & ns \\
\hline ESTADIO III & \multicolumn{2}{|c|}{$15 / 76$} & \multicolumn{2}{|c|}{$3 / 19$} & ns \\
\hline Aneuploides & \multicolumn{2}{|c|}{$46 / 60$} & \multicolumn{2}{|c|}{$12 / 16$} & ns \\
\hline $\mathrm{FS}>15,4 \%+{ }^{*}$ & \multicolumn{2}{|c|}{$28 / 49$} & \multicolumn{2}{|c|}{$3 / 13$} & 0,041 \\
\hline $\mathrm{FS}>25,4 \%+^{* *}$ & \multicolumn{2}{|c|}{$13 / 49$} & \multicolumn{2}{|c|}{$2 / 13$} & ns \\
\hline
\end{tabular}

$\mathrm{fmol} / \mathrm{mg}$ prot. ${ }^{*} ; \mathrm{NHI} / \mathrm{mg}$ prot. ${ }^{* *} ; \%++; \mathrm{cm}+++; \mathrm{FS}$ : fase de síntesis celular; GH: grado histológico; FS: fase de síntesis celular; Mediana de todo el grupo tumoral+*; Percentil 75 de todo el grupo tumoral $+{ }^{* *}$

en nitrógeno líquido hasta su utilización. Posteriormente, fueron pulverizadas con un microdesmembrador (OMNI 1000, Waterbury, CT. USA) a $-70^{\circ} \mathrm{C}$ y homogeneizadas en un potter de teflon -cristal en un buffer TRIS-CLH (TRIS 0,01 M, EDTA Na2 0,0015 M, 10\% glycerol, 0,1\% monothioglycerol, pH 7,4). Los homogeneizados fueron luego centrifugados a bajas revoluciones ( $800 \mathrm{~g}$ durante 10 minutos a $4^{\circ} \mathrm{C}$ ) y el sobrenadante obtenido ultracentifugado a $100,000 \mathrm{~g}$ durante una hora a $4^{\circ} \mathrm{C}$. El nuevo sobrenadante (citosol) y el precipitado (membranas) se utilizaron para los distintos análisis biológicos.

El CD44v6 fue determinado mediante un EIA (Bender Diagnostics. Austria). El límite inferior de sensibilidad se estableció en 0,13 ng/mL; los coeficientes de variación intraensayo para unos valores medios de 0,52 y $6,4 \mathrm{ng} / \mathrm{mL}$ fueron del $8,6 \%$ y $5,1 \%$ respectivamente. Los coeficientes de variación interensayo para unos valores medios de 3,47 y $9,2 \mathrm{ng} / \mathrm{mL}$ fueron del $9,3 \%$ y $8,4 \%$ respectivamente. El dintel de positividad fue establecido en $5 \mathrm{ng} / \mathrm{mg} \mathrm{prot}^{19}$. Otros parámetros analizados en las membranas celulares fueron las concentraciones del receptor del factor de crecimiento epidérmico (EGFR, Radioligando, Viennalab. Austria) y proteína oncogénica erbB2 (enzimoinmunoanálisis (EIA). Dupont. EEUU). Los valores de los parámetros biológicos fueron expresados por mg de proteína determinada por el método de Bradford. También se ha tenido presente el estadio clínico, grado histológico $(\mathrm{GH})$, ploidía y fase $S(\mathrm{FS})$, determinadas estas dos últimas mediante citometría de flujo (Becton Dickinson. Fascam. EEUU) en muestras en fresco. Como las concentraciones de los parámetros biológicos no siguieron una distribución gaussiana, hemos empleado el test de MannWhitney, así como el chi cuadrado (x2), con la corrección de Yates cuando fue necesaria, para la comparación de variables cualitativas. Los resultados han sido expresados mediante la mediana y el intervalo. Una diferencia se consideró estadística cuando el valor de $\mathrm{p}$ fue inferior a 0,05 .

\section{Resultados}

En los carcinomas escamosos de pulmón, la positividad para el CD44v6 fue apreciada en 76 de los 95 casos $(80 \%)$ y no difirió estadísticamente de la constatada en los tejidos pulmonares aparentemente normales de los mismos pacientes $(33 / 38 ; 86,8 \%)$. Sin embargo, sí observamos diferencias significativas ( $p: 0,0071$ ) entre las concentraciones de CD44v6 obtenidas en los tumores (i: 3.6-1305; mediana 47,5 ) y el tejido normal (i: 5-100; mediana 31, ng/mg prot.).

Los tumores CD44v6 positivos presentaron, tal como se expone en la Tabla I, mayores concentraciones de EGFR (p:0,007), proteína erbB2 (p:0,056) y valores 


\section{A. Ruibal y cols.}

globales de FS (p:0,011). Asimismo, cursaron más frecuentemente con $\mathrm{FS}+(>15,4 \%$, que representa la mediana obtenida en todo el grupo tumoral) $(p: 0,041)$.

\section{Discusión}

La utilidad clínica de la expresión de CD44v6 varía según el tumor que consideremos. Menos trabajos existen en relación con su comportamiento en los carcinoma pulmonares no microcíticos y por ello hemos querido estudiar, mediante un método inmunoenzimático, su concentración en las membranas celulares de muestras correspondientes a carcinomas escamosos. Usando como dintel de positividad una concentración de $5 \mathrm{ng} / \mathrm{mg}$ prot., similar al empleado en los carcinomas mamarios, pudimos apreciar expresión de la isoforma en el $80 \%$ de los casos, siendo superior a la obtenida en los adenocarcinomas pulmonares $157,6 \%$; datos no mostrados) y parecida a la observada en los tejidos pulmonares normales de los pacientes con aquel subtipo histológico de pulmón. Nuestra positividad tumoral es similar a la descrita por otros grupos ${ }^{20}$ ${ }^{21}$ y lo mismo ocurre con la observada en el tejido pulmonar aparentemente normal ${ }^{22}$. Aunque no constatamos diferencias en la expresión de CD44v6 entre tejido tumoral y normal, si pudimos apreciar mayores concentraciones de la isoforma en las muestras neoplásicas, lo cual sugiere un posible papel de aquella molécula en la transformación neoplásica.

Los carcinomas escamosos CD44v6 positivos mostraron mayores concentraciones de EGFR y proteína erbB2, y fueron, asimismo, más proliferativos (mayores valores de fase S).

La expresión de EGFR es mayor en los carcinomas escamosos (79\%) que en los otros tipos histológicos y superior a la constatada en el tejido pulmonar nor$\mathrm{mal}^{23}$. No se correlaciona con otros parámetros clínicos, aunque se han descrito mayores concentraciones en los casos con afectación ganglionar ${ }^{24}$. Su valor como factor pronóstico no es unánimemente aceptado por todos los grupos ${ }^{25}$, si bien otros autores consideran que es un indicador de un peor pronóstico y respuesta a la terapia ${ }^{25-27}$. Merece destacarse que parece ser la fosforilación y no la sobreexpresión del receptor la que se asocia con un peor comportamiento en los carcinomas no microcíticos de pulmón ${ }^{28}$. Piyathilake y cols. ${ }^{29}$ observan que la localización del EGFR en el citoplasma, pero no en la membrana, sí se asociaba con un peor pronóstico en los carcinomas escamosos y ello puede ser explicado por la internalización del receptor tras su unión al ligando.

En relación con el oncogen erbB2, se sabe que el epitelio normal no suele expresarlo, pero sí el 19-27\% de los tumores no microcíticos pulmonares, especialmente poco o moderadamente diferenciados. Su expresión es mayor en los adenocarcinomas que en escamosos y no parece ser factor pronóstico ${ }^{30}$, si bien otros grupos observan una relación con la afectación ganglionar y una tendencia a una menor superviven$\mathrm{cia}^{31,32}$. La positividad para este oncogen y el EGFR parece definir un subgrupo de tumores con un peor comportamiento y evolución ${ }^{33}$. Asimismo, este gen se correlaciona con la densidad de microvasos ${ }^{34}$ y es responsable de la resistencia a ciertos fármacos ${ }^{35}$.

Los carcinomas escamosos son más proliferativos que los adenocarcinomas, lo cual puede explicar una mayor agresividad y una mejor respuesta al tratamiento ${ }^{36}$. Asimismo, la proliferación tiene interés pronósti$\mathrm{co}^{37-40}$, de tal manera que en los tumores no microcíticos una menor proliferación y una mayor apoptosis se asocia con una superior supervivencia ${ }^{41}$.

Los resultados anteriores nos inducen a las siguientes consideraciones: 1) es posible detectar la expresión de CD44v6 en un alto porcentaje de los carcinomas escamoso de pulmón, no difiriendo de la observada en muestras aparentemente normales de los mismos pacientes; 2) las concentraciones de CD44v6 son mayores en los tumores que en los tejidos aparentemente normales de los mismos pacientes; 3) la positividad para el CD44v6 no se correlacionó con ninguno de los parámetros clínicos habituales, pero sí con mayores concentraciones de EGFR y proteína erbB2, así como una mayor proliferación celular. Aunque ello podría reflejar su relación con un peor comportamiento y evolución, en el momento actual no podemos precisar como la expresión de esta isoforma puede influir en el seguimiento de este subtipo histológico pulmonar.

Correspondencia:

Dr. A. Ruibal Morell

Servicio de Medicina Nuclear Hospital Clínico

Complejo Hospitalario Universitario

E-15706 Santiago de Compostela 


\section{Bibliografía}

1. Braunmuller H, Gansauge S, Ramadani M, Gansauge F. CD44v6 cell surface expression is a common feature of macrophages and macrophage-like cells-implication for a natural macrophage extravasation mechanims mimicked by tumor cells. FEBS Lett 2000; 476:240-7.

2. Kanke $M$, Fujii $M$, Kameyama K, Kanzaki J, Tokumaru $Y$, Imanishi $Y$, et al. Role of CD44 variant exon 6 in invasion of head and neck squamous cell carcinoma. Arch Otolaryngol Head Neck Surg 2000; 126:1217-23.

3. Yamaguchi A, Goi T, Yu J, Hirono Y, Ishida M, lida A, et al. Expression of CD44v6 in advanced gastric cancer and its relationshio to hematogenous metastasis and long-term prognosis. J Surg Oncol 2002; 79:230-5.

4. Ayhan A, Tok EC, Bildirici I, Ayhan A. Overexpression of CD44 variant 6 in human endometrial cancer and its prognostic significance. Gynecol Oncol 2001; 80:355-8.

5. Costa S, Terzano P, Bovicelli A, Martoni A, Angelelli B, Santini D, et al. CD44 isoform 6 (CD44v6) is a prognostic indicator of the response to neoadjuvant chemotherpay in cervical carcinoma. Gynecol Oncol 2001; 80:6773.

6. Ristamaki R, Joensuu $H$, Soderstrom KO, Jalkanen S. CD44v6 expression in non-Hodgkin's lymphoma: an association with low histological grade and poor prognosis. J Pathol 1995; 176:259-67.

7. Maula S, Huuhtanen RL, Blomqvist CP, Wiklund TA, Laurila $P$, Ristamaki $R$. The adhesion molecule CD44v6 is associated with a high risk for local recurrence in adult soft tissue sarcomas. Br J Cancer 2001; 84:244-52.

8. Saito H, Tsujitami S, Katano K, Ikeguchi M, Maeta M, Kaibara N. Serum concentration of CD44 variant 6 and its relation to prognosis in patients with gastric carcinoma. Cancer 1998; 83:1094-101.

9. Yamane N, Tsujitami S, Makino M, Maeta M, Kaibara $N$. Soluble CD44 varinat 6 as a prognostic indicator in patients with colorectal cancer. Oncology 1999; 56: 232-8.

10. Gansauge F, Gansauge S, Rau B, Scheiblich A, Poch B, Schoenberg $M H$, et al. Low serum levels of soluble CD44 variant 6 are significantly associated with poor prognosis in patients with pancreatic carcinoma. Cancer 1997; 80:1733-9.

11. Kopp R, Classen S, Wolf H, Gholam P, Possinger K Wilmanns W. Predictive relevance of soluble CD44v6 serum levels for the responsiveness to second line-hormone or chemotherapy in patients with metastatic breast cancer. Anticancer Res 2001; 21:2995-3000.

12. Leir SH, Baker JE, Holgate ST, Lackie PM. Increased CD44 expression in human bronchial epithelial repair after damage or plating at low cell densities. Am J Physiol Lung Cell Mol Physiol 2000; 278: L1 129-37.

13. Ylagan LR, Scholes J, Demopoulos R. CD44: a marker of squamous differentiation in adenosquamous neoplasms. Arch Pathol Lab Med 2000; 124:21 2-5.
14. Kasper M, Gunther U, Dall P, Kayser K, Schuh D, Haroske $G$, et al. Distinct expression patterns of CD44 isoforms during human lung development and pulmonary fibrosis. A,m J Respir Cell Moll Biol 1995; 13:648-56.

15. Fasano M, Sabatini MT, Wieczorek R, Sidhu G, Goswami S, Jagirdar J. CD44 and its v6 spliced variant in lung tumors: a role in histogenesis? Cancer 1997; 80:34-41.

16. Coppola D, Clarke M, Lanmdreneau R, Weyant RJ, Cooper D, Yousem SA. Bcl-2, p53, CD44 and CD44v6 isoform expression in neuroendocrine tumors of the lung. Mod Pathol 1996; 9:484-90.

17. Miyoshi T, Kondo K, Hino N, Uyama T, Monden Y. The expression of the CD44 variant exon 6 is associated with lymph node metastasis in non-small cell lung cancer. Clin Cancer Res 1997; 3:1 289-97.

18. Tran TA, Kallakury BV, Sheehan CE, Ross JS. Expression of CD44 standard form and variant isoforms in nonsmall cell lung carcinomas. Hum Pathol 1997; 28:80914.

19. Ruibal A, Schneider J, del Río MC, Arias Jl, Núñez MI, Tejerina A. La expresión de la molécula de adhesión CD44v6 en carcinomas ductales infiltrantes de mama se asocian con la hormonodependencia. Nuestra experiencia con 168 casos. Rev Esp Med Nuclear 2000; 19:350-5

20. Nguyen VN, Mirejovsky T, Melinova L, Mandys V. CD44 and its v6 spliced variant in lung carcinomas: relation to NCAM, CEA, EMA and UP1 and prognostic significance. Neoplasma. 2000; 47:400-8.

21. Ariza A, Mate JL, Isamat $M$, Lopez D, Von Uexkull-Guldeband C, Rosell R, et al. Standard and variant CD44 isoforms are commonly expressed in lung cancer of the non-small cell type but not of the small cell type. J Pathol. 1995; 177:363-8.

22. Shimbori $M$, Kijima $H$, Sato $S$, Yoshida $H$, Sato T, Terasaki-Fukuzawa $Y$, et al. Expression of CD44 in primary lung carcinomas using histological and cytological analyses. Anticancer Res. 2003 Jan-Feb; 23(1A): 1 15-21.

23. Díez M, Maestro ML, Torres A, Hernando F, Ortega MD, García-Asenjo JA, et al. El receptor del factor de crecimiento epidérmico en carcinomas no microcíticos de pulmón. Arch Bronconeumol. 1994 Jun-Jul; 30(6): 282-6.

24. Fontanini G, Vignati S, Bigini D, Mussi A, Luchi $H$, Angeletti CA, et al. Epidermal growth factor receptor (EGF$\mathrm{R})$ expression in non-small cell lung carcinomas correlates with metastatic involvement of hiliar and mediastinal lymph nodes in the squamous subtype. Eur J Cancer $1995 ; 31: 178-83$.

25. Penault-Llorca F, Durando X, Bay JO. Prognostic value of epidermal growth factor receptor. Bull Cancer 2003; 90:S192-6.

26. Volm $M$, Drings $P$, Wodrich $W$. Prognostic significance of the expression of c-fos, c-jun and c-erbbl oncogene products in human squamous cell lung carcinomas. J Cancer Res Clin Oncol 1993; 119:507-10. 
27. Selvaggi G, Novello S, Torri V, Leonardo E, De Giuli P, Borasio $P$, et al. Epidermal growth factor overexpression correlates with a poor prognosis in completely resected non-small cell lung cancer. Ann Oncol 2004; 15:28-32.

28. Kanematsu T, Yano S, Uehara H, Bando $Y$, Sone $S$. Phosphorylation, but not overexpression, of epidermal growth factor receptor is associated with poor prognosis of non-small cell lung cancer patients. Oncol Res 2003; 13:289-98.

29. Piyathilake CJ, Frost AR, Manne U, Weiss H, Bell WC, Heimburger DC, et al. Differential expression of growth factors in squamous cell carcinoma and precancerous lesions of the lung. Clin Cancer Res 2002; 8:734-44.

30. Moldvay J, Scheid P, Wild P, Nabil K, Siat J, Borrelly J, et al. Predictive survival markers in patients with surgically resected non-small cell lung carcinoma. Clin Cancer Res 2000; 6:1 1125-34.

31. Volm M, Koomagi R, Mattern J, Efferth T. Protein expression profile of primary human squamous cell lung carcinomas indicative of the incidence of metastases. Clin Exp Metastasis 2002; 19:385-90.

32. Tan D, Deeb G, Wang J, Slocum HK, Winston J, Wiseman $S$, et al. HER-2/neu protein expression and gene alteration in stage I-IIIA non small cell lung cancer: a study of 140 cases using a combination of high throughput tissue microarray, immunohistochemistry, and fluorescent in situ hybridization. Diagn Mol Pathol 2003; 12:201-11.

33. Onn A, Correa AM, Gilcrease M, Isobe T, Massarelli E, Bucana $C D$, et al. Synchronous overexpression of epidermal growth factor receptor and HER-2 neu protein is a predictor of poor outcome in patients with stage I nonsmall cell lung cancer. Clin Cancer Res 2004; 10:13643.
34. Brattstrom D, Wester $K$, Bergqvist $M$, Hasselius $P$, Malmstrom PU, Nordgren $\mathrm{H}$, et al. HER-2, EGFR , COX2 expression status correlated to microvessel density and survival in resected non-small cell lung cancer. Acta Oncol 2004; 43:80-6.

35. Higashiyama M, Doi O, Kodama K, Yokouchi H, Kasugai $T$, Ishiguro $S$. Influence of cathepsin $D$ expression in lung adenocarcinoma on prognosis : possible importance of its expression in tumor cells and stromal cells, and its intracellular polarization in tumor cells. J Surg Oncol 1997; 65:10-9.

36. Mangili F, Cigala C, Arrigoni G, Rovere E, Gattuso C, Santambrogio $G$, et al. Cell loss and proliferation in non-small cell lung carcinoma: correlation with histological subtype. Eur J Histochem 1998; 42:287-95.

37. Pelletier MP, Edwards MD, Michel RP, Halwani F, Morin JE. Prognostic markers in resected non-small cell lung cancer: a multivariate analysis. Can J Surg 2001; 44:180-8.

38. Dobashi Y, Jiang SX, Shoii M, Morinaga S, Kameya T. Diversity in expression and prognostic significance of G1/S cyclins in human primary lung carcinomas. J Pathol 2003; 199:208-20.

39. Caputi M, Espósito V, Groger AM, Pacilio C, Murabito $M$, Dekan $G$, et al. Prognostic role of proliferating cell nuclear antigen in lung cancer: an immunohistochemical analysis. In Vivo 1998; 12:85-8.

40. Alvarez Riesgo JA, Sampedro A, Hernández R, Folgueras $M V$, Salas-Bustamante A, Cueto A. Cell proliferative activity and prognostic index in squamous cell lung carcinoma. Anal Cell Pathol 1998; 16:233-42.

41. Mattern J, Koomagi R, Volm M. Characteristics of longterm survivors of untreated lung cancer. Lung Cancer 2002; 36:277-82. 